\title{
Un dossier disciplinaire dans une revue interdisciplinaire : pourquoi ?
}

La revue NSS publie de plus en plus de dossiers thématiques. Elle répond ainsi à une demande croissante de la communauté scientifique, qui voit en NSS un support pour rendre compte de débats engageant plusieurs disciplines, parfois à la suite d'un colloque ou d'un séminaire, parfois à l'occasion d'échanges qui se construisent dans la durée, par exemple autour d'un programme de recherche appelant au dialogue interdisciplinaire. NSS n'a pas vocation à ne s'exprimer que sous la forme de ces dossiers, mais leur présence, en augmentation dans la revue, n'est pas fortuite. Les modalités d'un travail scientifique de plus en plus cadré par des programmations de recherche, et dont l'aboutissement emprunte le plus souvent les voies de manifestations collectives, nous y incitent fortement. C'est aussi une volonté éditoriale de conserver à la livraison d'un numéro une unité que mettent à mal la mise en ligne et l'usage qui en découle.

Dans ce numéro, la publication du dossier sur l'économie de la proximité relève de l'établissement d'un état de l'art disciplinaire. En cela, il fait exception. Mais qu'est-ce que l'économie de la proximité et pourquoi ce dossier vous est-il proposé?

L'économie de la proximité est un courant de pensée qui s'est construit au sein de l'économie, à l'intersection de l'économie spatiale et de l'économie industrielle, et qui tire son originalité de la façon dont il analyse les phénomènes territoriaux dans les processus économiques. Son principal apport est d'avoir renouvelé l'appréhension de la notion de distance par la mise en tension des phénomènes spatiaux et des interactions entre acteurs, à travers le couple conceptuel proximité géographique / proximité organisée. Il se situe dans la lignée de l'économie industrielle et de l'approche évolutionniste qui ont alimenté de longue date l'économie régionale ; mais, dans le même temps, il se positionne à la frontière de problématiques abordées par d'autres disciplines. Son point de vue sur la question territoriale ne pouvait ignorer les problèmes d'environnement, dont l'une des dimensions est la marque qu'ils impriment sur les territoires, marque qui, en retour, en conditionne les formes et les modalités de recomposition.

NSS a donc accepté de publier ce dossier, soumis par un groupe d'économistes animé par André Torre et Bertrand Zuindeau, avec un double objectif. Il s'agissait d'abord d'être à l'écoute d'approches peu représentées jusqu'alors dans nos colonnes et surtout de mettre à l'épreuve les concepts de leur courant de pensée avec les questions scientifiques que pose l'environnement et qui sont également abordées par d'autres communautés scientifiques. Le mandat était le suivant : les deux porteurs du dossier proposaient un cadrage théorique et problématique rendant compte de l'intérêt d'une telle rencontre et plusieurs contributions centrées sur différents cas d'étude illustraient la capacité de l'économie de la proximité à investir le champ de l'environnement. D'une certaine façon, il s'agissait d'évaluer les acquis, les atouts, mais aussi les limites de ce courant pour traiter des problèmes d'environnement et, en retour, d'identifier ce qu'il était susceptible de nous apprendre des objets et des problèmes analysés en partant de leur proposition paradigmatique.

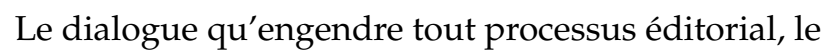
nôtre faisant appel à des lecteurs légitimes pour discuter des thèses avancées, a été particulièrement enrichissant. En quoi l'approche par l'économie de la proximité renouvelle-t-elle l'analyse des conflits environnementaux qu'abordent également d'autres disciplines? Qu'est-cequi différencie, par exemple, des conflits environnementaux ceux qu'analyse l'économie de la proximité en distinguant les conflits qui se situent dans une proximité géographique et ceux qui s'inscrivent dans une proximité plus lointaine qualifiée d'organisée? Peut-on aussi aisément dissocier le proche et le lointain, avec les propriétés qui, pour eux, leur sont attachées - l'exaspération d'un côté et l'apaisement de l'autre, le conflit irréductible d'un côté et la construction de l'accord de l'autre-, alors que les questions d'environnement, du fait des interdépendances 
qu'elles révèlent ou exacerbent, brouillent de telles catégories et appellent une approche territoriale où la dimension physique relève moins des effets de distance et davantage des propriétés multiformes - et complexes - du territoire? En posant ces questions aux économistes de la proximité, nous les avons incités à clarifier ce qu'ils entendent par environnement, car chacun sait qu'il peut abriter de multiples objets et de nombreuses acceptions. De la même façon, en convoquant dans leur démarche la question de l'incertitude, qui est la marque de nombreux problèmes d'environnement, ils ont mesuré tout à la fois les obstacles théoriques à un tel investissement et le bénéfice qu'ils peuvent en tirer pour proposer des outils conceptuels en phase avec les enjeux de la modernité.

Le pari est-il tenu, que ce soit du point de vue des responsables du dossier ou de celui du comité de rédaction? $\mathrm{Au}$ lecteur d'en juger. Nous pouvons seulement souhaiter que le dialogue ou la controverse s'instaurent à la faveur de cette livraison, pour laquelle l'interdisciplinarité n'est pas au centre du propos, mais qui appelle la confrontation avec d'autres disciplines, du droit à la sociologie en passant par la géographie. Elles partagent de fait un certain nombre de concepts ou de questions avec ce courant de l'économie. Mais ce dialogue doit aussi s'instaurer au sein même de l'économie, en particulier celle qui se revendique d'une hétérodoxie - économie de l'environnement, de la régulation, des conventions, etc. - que peut interroger l'économie de la proximité dans son souci de mettre la question territoriale au cœur de l'approche économique. Et cette économie hétérodoxe peut aussi interpeller l'économie de la proximité dans sa capacité à s'affranchir, dans son analyse des stratégies d'acteurs, par exemple, d'une rationalité limitée à celle de l'intérêt que définit le « coût-avantage». De ce point de vue, la problématique environnementale attend de l'économie de la proximité qu'elle inscrive résolument son projet dans une économie politique élargie, pour le moins celle que promeut Paul Krugman, prix Nobel d'économie en 2008, à la fois source de renouveau de la pensée économique sur l'espace et objet de critique pour sa focalisation excessive sur les seules règles du marché.

Au moment où le prix Nobel 2009 a été attribué à Elinor Ostrom - événement dont la revue se fera l'écho dans un futur numéro -, on ne peut que souligner l'importance du dialogue entre disciplines. La trajectoire couronnée est en effet celle d'un parcours intellectuel qui a constamment transgressé les frontières disciplinaires, ce à quoi NSS tente de contribuer - avec modestie, certes, mais avec la certitude que ce projet éditorial est plus que jamais essentiel. La proposition de ce dossier sur l'économie de la proximité nous convainc que le franchissement de telles frontières n'est pas aisé, mais qu'il est de notre rôle de l'accompagner et même de l'encourager. Ce courant interpelle d'autres disciplines auxquelles il emprunte des concepts ou des méthodes pour les retravailler et avec lesquelles il instaure de fait des rapports de voisinage. C'est pourquoi la publication de ce dossier centré sur un courant disciplinaire n'a de sens que parce qu'il est aussi une invite au débat entre disciplines.

Jean-Paul Billaud, Daniel Terrasson 